\title{
Preparation and Characterization of Porous Scaffold Composite Films by Blending Carboxymethyl Chitosan and Gelatin for Tissue Engineering
}

\author{
Nisat Tamanna Nipu ${ }^{1}$, Farzana Khan Rony ${ }^{2}$, Asaduz Zaman ${ }^{1,}$ * \\ ${ }^{1}$ Department of Applied Chemistry and Chemical Engineering, University of Dhaka, Dhaka, Bangladesh \\ ${ }^{2}$ Institute of Glass and Ceramic Research and Testing, Bangladesh Council of Scientific and Industrial Research, Dhaka, Bangladesh \\ Email address: \\ asad.acce@du.ac.bd(A.Zaman) \\ ${ }^{*}$ Corresponding author
}

\section{To cite this article:}

Nisat Tamanna Nipu, Farzana Khan Rony, Asaduz Zaman. Preparation and Characterization of Porous Scaffold Composite Films by Blending Carboxymethyl Chitosan and Gelatin for Tissue Engineering. International Journal of Materials Science and Applications. Vol. 7, No. 2, 2018, pp. 62-68. doi: 10.11648/j.ijmsa.20180702.15

Received: February 23, 2018; Accepted: March 9, 2018; Published: April 2, 2018

\begin{abstract}
In this research work, gelatin-carboxymethylchitosan (CMC) based biodegradable composites films were prepared by solution casting method. Chitosan from waste prawn shell was the basic raw materials of CMC synthesis. Five sets of CMC-gelatin composites (5-25 wt \% CMC) along-with pure gelatin were prepared in solution casting method. Incorporation of $\mathrm{CMC}$ into gelatin significantly altered some of the properties. The CMC and gelatin-CMC composites formation was confirmed by Fourier Transform Infrared Spectroscopy (FTIR). Surface morphology of the films was investigated by Scanning Electron Microscopy (SEM) and SEM micrograph revealed that composites were porous and CMC was homogenously dispersed into gelatin. The porous surface of the composites is one of the criterions for new cells growth. Thermal stability of composites were investigated by thermogravimetric analysis (TGA) and composites more thermal stable (less weight loss) than pure gelatin. Antimicrobial and cytotoxicity tests found all composites were performed microbial safe and no cytotoxic effect. The physico-chemical analyses and others analyses of scaffolds revealed for their application as a wound dressing material or artificial skin.
\end{abstract}

Keywords: Carboxymethyl Chitosan, Gelatin, Scaffold, Cytotoxicity and Tissue Engineering

\section{Introduction}

The tissue engineering approach to repair and regeneration is founded upon the use of polymer scaffolds which serve to support, reinforce and in some cases organize the regenerating tissue [1-3]. Scaffolds are among the key components of a tissue-engineered construct and affect the healing process, forming an environment for the cells similar to that of extracellular matrix (ECM). Natural polymers have great resemblance to natural ECM elements, especially in biocompatibility and biodegradability, and have therefore gained much attention as scaffold materials [4]. A number of natural and synthetic polymers are currently being employed as tissue scaffolds. The microstructures of these systems span the range from hydrogels, to open-pore structures, to fibrous matrices [5-7]. Since the range of potential tissue engineered systems is broad, there is a continuous ongoing search for materials which either possess particularly desirable tissuespecific properties, or which may have broad applicability and can be tailored to several tissue systems [1].

Gelatin is a soluble protein derived from partially denatured collagen. Attractive properties of gelatin, such as good biocompatibility, low immunogenicity, plasticity, adhesiveness, promotion of cell adhesion and growth, and low cost, make it ideally suitable as a biomaterial for tissue engineering. Gelatin contains free carboxyl groups on its backbone and has the potential to blend with chitosan and chitosan derivatives to form a network by hydrogen bonding $[8,9]$. Chitosan, a linear polysaccharide, is the Ndeacetylated derivative of chitin, holds great potential in tissue engineering applications due to its readily availability, unique physicochemical properties, biocompatibility and 
biodegradability [10]. It has been used for drug delivery, sutures, and skin repair. One of chitosan's most exceptional features is its ability to fabricated in the form of lyophilized porous scaffolds to support cell attachment and proliferation, and eventually to facilitate new tissue formation after chitosan degradation [11]. The decomposition rate of the chitosan used up to now as scaffolds is slow and uncontrollable. Chitosan has been restricted in many applications because one usually wishes to match the degradation rate of materials to the regeneration rate of new tissues [12]. Carboxymethyl chitosan (CMC), a dissolvable chitosan derivative, also possesses many desirable physiochemical and biological features, for instance gelating capability, nontoxicity, and biocompatibility [13]. CMC has an excellent degradable capability due to its solubility at physiological $\mathrm{pH}$ and appropriate materials for tissue regeneration scaffolds $[14,15]$. However, the details study of gelatin-CMC scaffolds has not been investigated yet.

In this study, carboxymethyl chitosan was synthesized from chitosan and complete characterized. Then gelatinCMC scaffolds were prepared by incorporating different loading CMCs with gelatin. The prepared scaffolds were performed physico-chemical, thermal and morphological characteristics. Finally, the suitability of the scaffold for human body compatibility was found out by performing in vitro degradation tests.

\section{Materials and Methods}

\subsection{Materials}

Chitosan was prepared from waste prawn shell. The viscosity average molecular weight and DD of extracted chitosan were $167,231 \mathrm{Da}$ and $84.4 \%$, respectively. The materials used for the synthesis of CMC is chitosan that was extracted from waste prawn shell and was collected from export oriented prawn farm located at the northern part Bangladesh. The analytical grade monochloroacetic acid, isopropanol, sodium hydroxide, hydrochloric acid and absolute ethanol were obtained from Merck Germany and used as received without any further purification.

\subsection{Methods}

\subsubsection{Preparation of Carboxymethyl Cellulose}

Carboxymethyl chitosan was synthesized from chitosan by using a modified method of Chen and Park [16]. The chitosan was suspended to swell in isopropanol at room temperature for overnight. The swell chitosan was refluxed with $40 \%(\mathrm{w} / \mathrm{w})$ at $85^{\circ} \mathrm{C}$ for $1.5 \mathrm{hrs}$. The mixture was refluxed again at $65^{\circ} \mathrm{C}$ for $4 \mathrm{hrs}$ under extensive stirring with drop-wise $40 \%$ (w/v) monochloroacetic acid and then neutralized with $5 \mathrm{~N} \mathrm{HCl}$. The dissolved carboxymethyl chitosan was precipitated in absolute ethanol, filtered and dried carefully below $60^{\circ} \mathrm{C}$. The dried $\mathrm{CMC}$ was stored in desiccator and further used in composite preparation.

\subsubsection{Preparation of Gelatin-CMC Scaffold}

Gelation-CMC composite films were prepared by mixing of $2 \%(\mathrm{w} / \mathrm{v}) \mathrm{CMC}$ solution and $10 \%(\mathrm{w} / \mathrm{v})$ gelatin solution. Five sets (gelatin to CMC ratio 95:5, 90:10, 85:15, 80:20, and $75: 25)$ of scaffold were cast in silicon sheet frame. Required amount of gelatin solution and CMC solution were mixed and formed homogeneous solution under constant agitation of magnetic stirrer at $50^{\circ} \mathrm{C}$ and then the solution was subjected to ultrasonication in order to remove bubbles. The gelatin-CMC solutions (80 $\mathrm{gm})$ were poured into silicon cloth glass dies $(3.5 \mathrm{~cm} \times 2.5 \mathrm{~cm})$ and placed in laminar flow for 24-30 hrs to form films. The dried films (about $0.25 \mathrm{~mm}$ thickness) were peeled off and cut into definite shape as required for the mechanical and thermal analysis and then preserved in the desiccators. A pure gelatin film was also cast in the same way.

\subsubsection{FTIR Analysis}

A Fourier transform infrared (FTIR) spectrophotomer (model: IRPrestige-21, Shimadzu Corporation, Japan) was used to investigate the characteristic peak of CMC, gelatin and gelatin-CMC scaffold within the range of 4000-700 $\mathrm{cm}^{-1}$.

\subsubsection{Thermal Analysis}

Thermogravimetric analysis (TGA) of the gelatin and gelatin-CMC composite films were carried out using a thermogravimetric analyzer (TGA-50, Shimadzu, Japan) at a heating rate of $10^{\circ} \mathrm{C} / \mathrm{min}$ within the range of $30-600^{\circ} \mathrm{C}$ using aluminum pan.

\subsubsection{Morphological Analysis}

The morphology composition of CMC, gelatin and gelatinCMC composites films were investigated using a highresolution scanning electron microscope (model no. JOEL JSM-6490 LA, Joel Ltd., Japan) instrument at $3.0 \mathrm{~nm}$ and an accelerating voltage of $10 \mathrm{kV}$. The non-conducting samples were set on a carbon tape to convert conducting film and placed to analysis.

\subsubsection{Cytotoxicity of Composites}

Cytotoxic analysis of gelatin-CMC composites was estimated following the standard method of Thi et al. [17]. In brief, HeLa, a human cervical carcinoma cell line was maintained in DMEM (Dulbecco's Modified Eagle's medium) containing $1 \%$ penicillin-streptomycin $(1: 1)$ and $0.2 \%$ gentamycin and $10 \%$ fetal bovine serum (FBS). Cells $\left(4 \times 10^{\wedge} 4 / 400 \mu \mathrm{l}\right)$ were seeded onto 24 -well plates and incubated at $37^{\circ} \mathrm{C}+5 \% \mathrm{CO}_{2}$. Next day $100 \mu$ of sample (autoclaved previously) was added at each well. Cytotoxicity was examined under an inverted light microscope after $24 \mathrm{~h}$ of incubation. Duplication wells were used for each sample.

\subsubsection{Antimicrobial Activity of Gelatin-CMC Composites (Agar Disk Diffusion Method)}

The antimicrobial activity of composites was determined for a selected gram positive bacteria (Staphylococcus aureus) and a gram negative bacteria (Escherichia coli) by the standard disk diffusion method [18]. Gram negative bacteria 
Escherichia coli and gram positive bacteria staphylococcus aureus were selected to assess susceptibility pattern and collected from the Food laboratory, CARS, University of Dhaka. The selected bacterial strains were cultured in the sterile tryptic soya broth solutions. The sterile tryptic soya agar (TSA) was placed in petri plates. The cultured bacterial strains were swabbed using cotton bud into the TSA plate and the sample films were injected within 15 mins after swabbing. Then the petri plates containing the sample and bacterial strain were incubated at $37^{\circ} \mathrm{C}$ for $18 \mathrm{~h}$ and then inhibition zone appeared for both samples. The measurements of the zones of inhibition were made with a ruler on the undersurface of the plate without opening the lid. The zones of growth inhibition were compared with standard drug loaded disks.

\section{Results and Discussion}

\subsection{FTIR Analysis of Chitosan, Carboxymethyl Chitosan and Gelatin-CMC Composites}

The FTIR spectra of chitosan and CMC are presented in Figure 1. The broad peak in CMC at $3400-3200 \mathrm{~cm}^{-1}$ is caused by both $\mathrm{O}-\mathrm{H}$ and $\mathrm{N}-\mathrm{H}$ stretching vibrations and the peak at $2900 \mathrm{~cm}^{-1}$ is due to the $\mathrm{C}-\mathrm{H}$ stretching vibrations. The spectra of CMC shows a strong peak at $1741 \mathrm{~cm}^{-1}$ and $1430 \mathrm{~cm}^{-1}$ which could be assigned to the $\mathrm{C}=\mathrm{O}$ stretching of carboxyl group and $\mathrm{C}-\mathrm{H}$ bending vibration of alkyl group. Both chitosan and CMC give strong peak at $1650-1630 \mathrm{~cm}^{-1}$ owing to $\mathrm{N}-\mathrm{H}$ bending of amine group. These results indicated that the carboxymethylation process had occurred at the $\mathrm{C}_{6}$ position of chitosan.

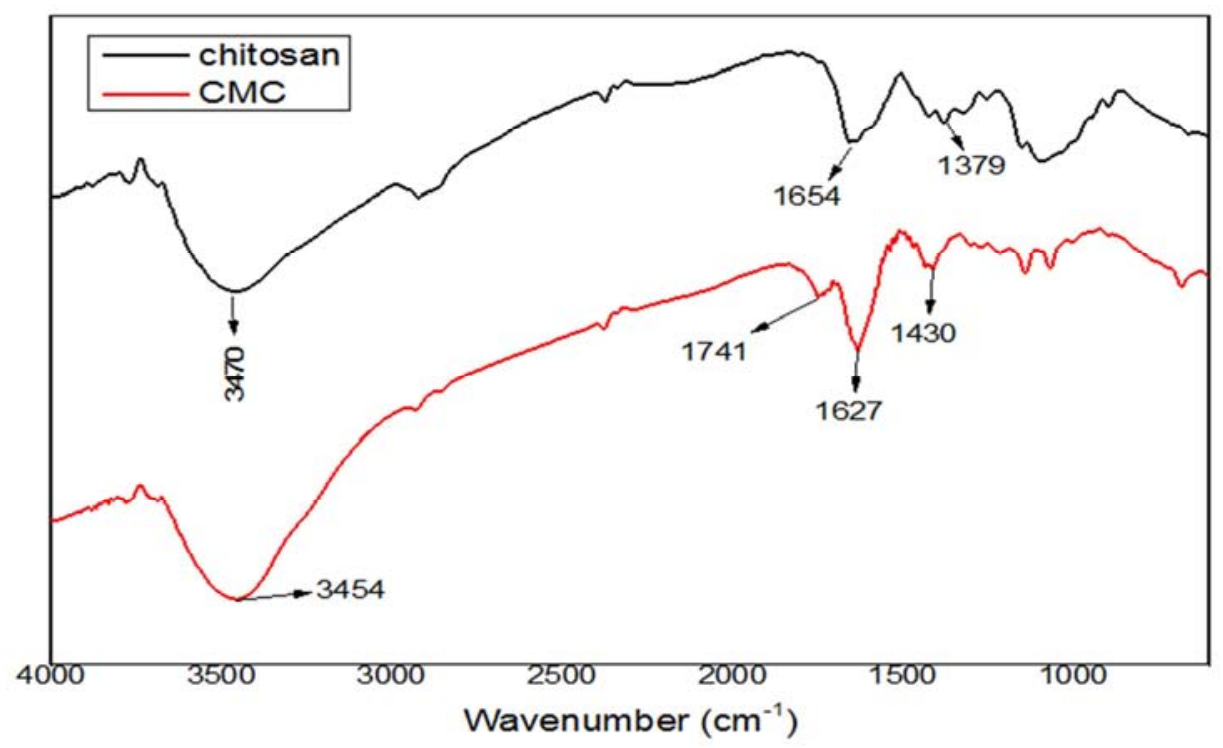

Figure 1. FTIR spectra of Chitosan and Carboxymethyl Chitosan.

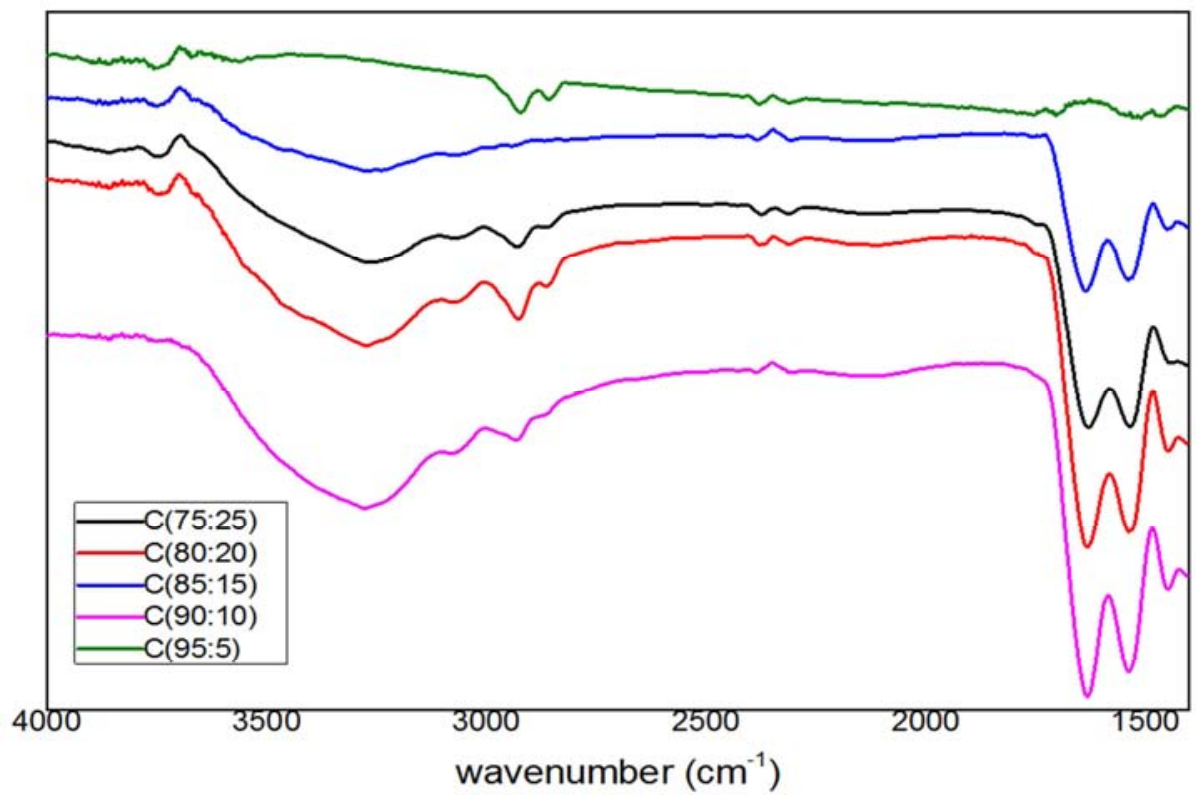

Figure 2. FTIR spectra of different CMC loaded gelatin composites. 
The IR spectra of gelatin-CMC composites are shown Figure 2. Peaks have been shifted from $3425 \mathrm{~cm}^{-1}$ (gelatin), $3456 \mathrm{~cm}^{-1}$ (CMC) to $3269 \mathrm{~cm}^{-1} ; 1604 \mathrm{~cm}^{-1}$ (gelatin) to $1635 \mathrm{~cm}^{-1}$. This modification can be explained by coupling of the anionic groups in $\mathrm{CMC}$ with the cationic ones in gelatin. The peak at $3269 \mathrm{~cm}^{-1}$ in the composite film is much broader than both gelatin and $\mathrm{CMC}$. Also the characteristic peak for $\mathrm{COOH}$ at $1749 \mathrm{~cm}^{-1}$ has been disappeared. In $1531 \mathrm{~cm}^{-1}$ a peak has appeared for amide bond formation between $\mathrm{COOH}$ of gelatin and $\mathrm{NH}_{2}$ of $\mathrm{CMC}$. Moreover, the peak of $\mathrm{C}-\mathrm{H}$ bending has been shifted from $1411 \mathrm{~cm}^{-1}$ to $1450 \mathrm{~cm}^{-1}$ which indicates the higher porosity in the composite [19].

\subsection{Morphological Analysis}

The scanning electron micrograph of pure gelatin, pure CMC, CMC-gelatin composites and fracture surface of CMC-gelatin composites are presented in Figure 3. From the micrograph, it is obvious that the both surfaces of gelatin and scaffold are uneven and less porosity. This feature dominated the surface of pure gelatin membrane. The pure $\mathrm{CMC}$ has higher porosity but the surface is less rough. However the gelatin-CMC scaffolds observed higher $\mathrm{CMC}$ ratio a much plainer texture with an incremented porosity. It is attributed that $\mathrm{CMC}$ could ameliorate the smoothness of the surface of gelatin film.
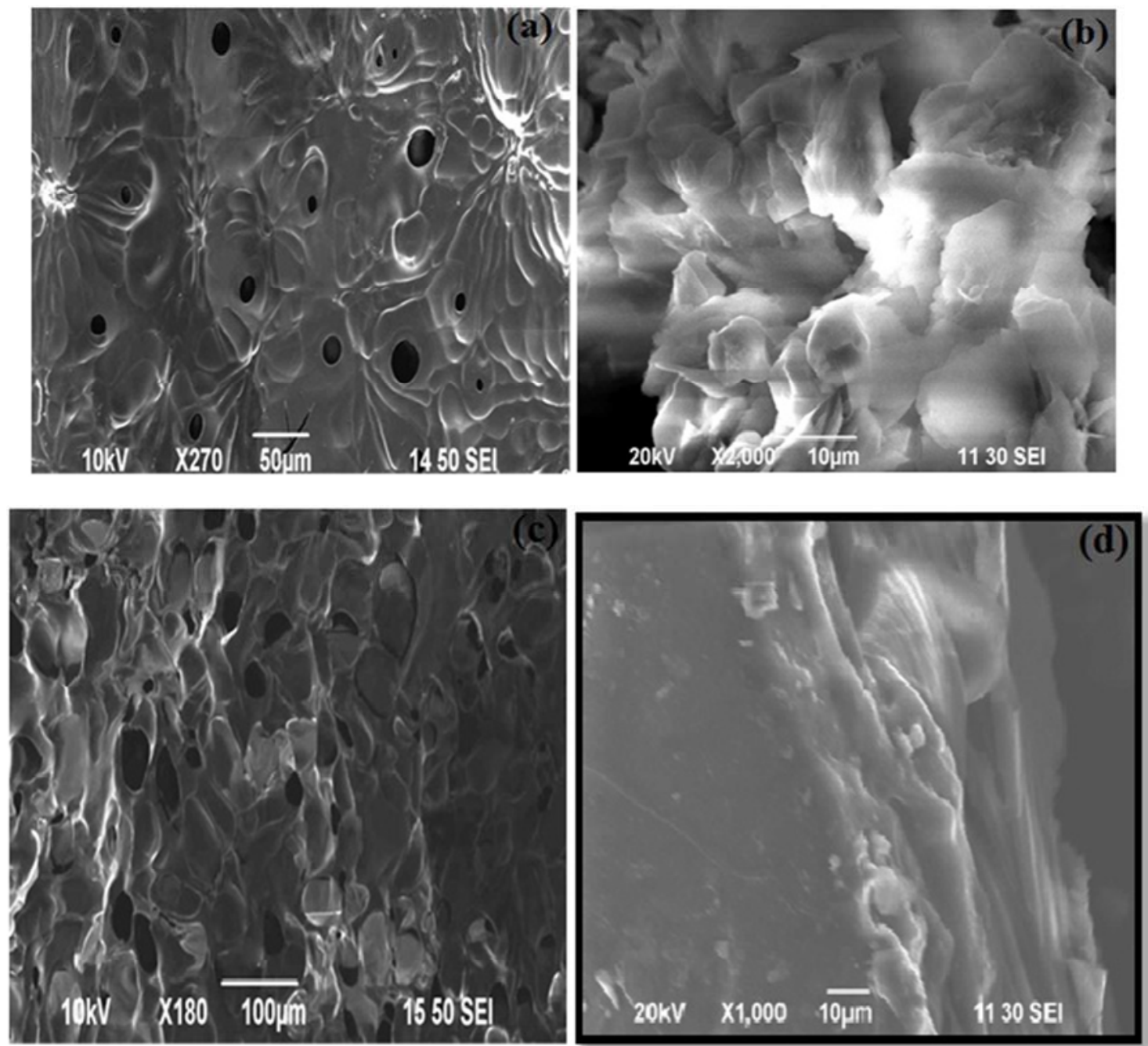

Figure 3. SEM image of (a) gelatin, (b) CMC, (c) Gelatin-CMC composite and (d) fractured cross section of composite.

\subsection{Thermal Analysis}

The TG thermograph of pure gelatin, pure $\mathrm{CMC}$, and CMC-gelatin composites are shown presented in Figure 4. From the thermograph, pure gelatin shows three zones of weight loss. The first weight loss at $268.97^{\circ} \mathrm{C}$ was due to the loss of water; the second weight loss was at $341.84^{\circ} \mathrm{C}$ for gelatin decomposition, and third weight loss was at $503.69^{\circ} \mathrm{C}$, showing that thermal degradation of gelatin took place and the total degradation is $90.03 \%$ [20]. Pure CMC shows two zones of weight loss. The first weight loss at $251.42^{\circ} \mathrm{C}$ was due to the loss of water; the second weight loss was at $349.25^{\circ} \mathrm{C}$, showing that thermal degradation of CMC took place and the total degradation is $55.15 \%$. From the thermograph it is clear that degradation of gelatin is highest and $\mathrm{CMC}$ is lowest. With the addition of $\mathrm{CMC}$, degradation of the composite film decreases and higher concentration of CMC composites are more stable than pure gelatin. This is due to the intermolecular interaction between gelatin and $\mathrm{CMC}$. 


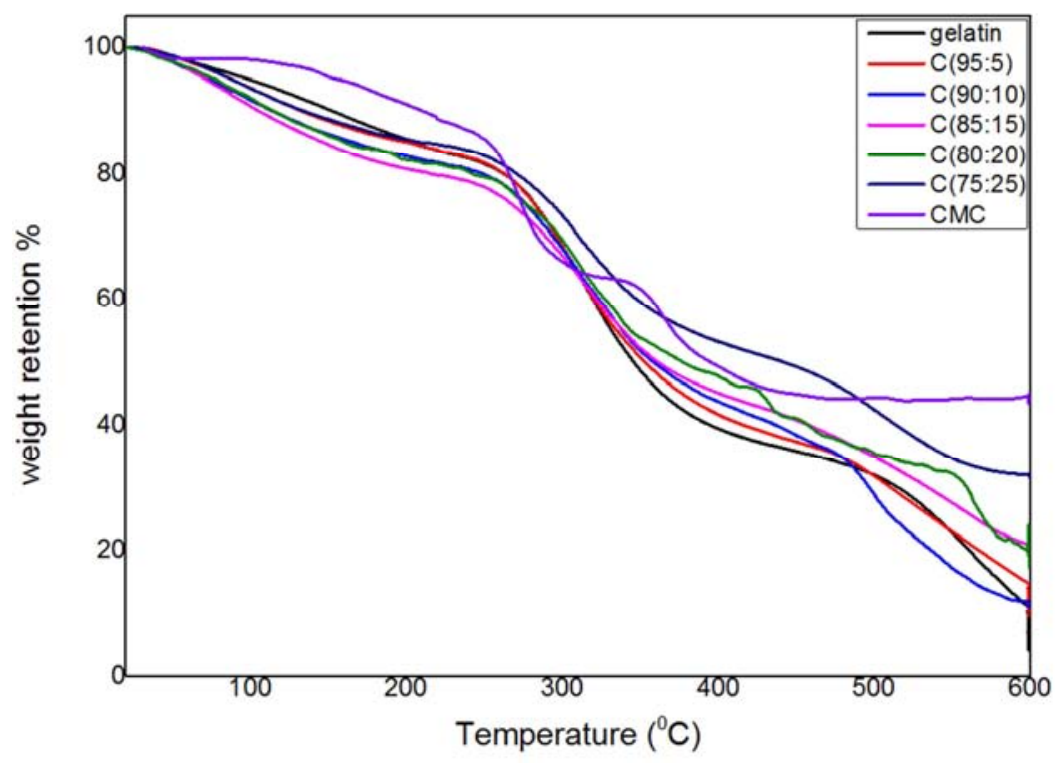

Figure 4. TG thermograph of gelatin, CMC and CMC-gelatin composites.

\subsection{Cytotoxic Effect Analysis}
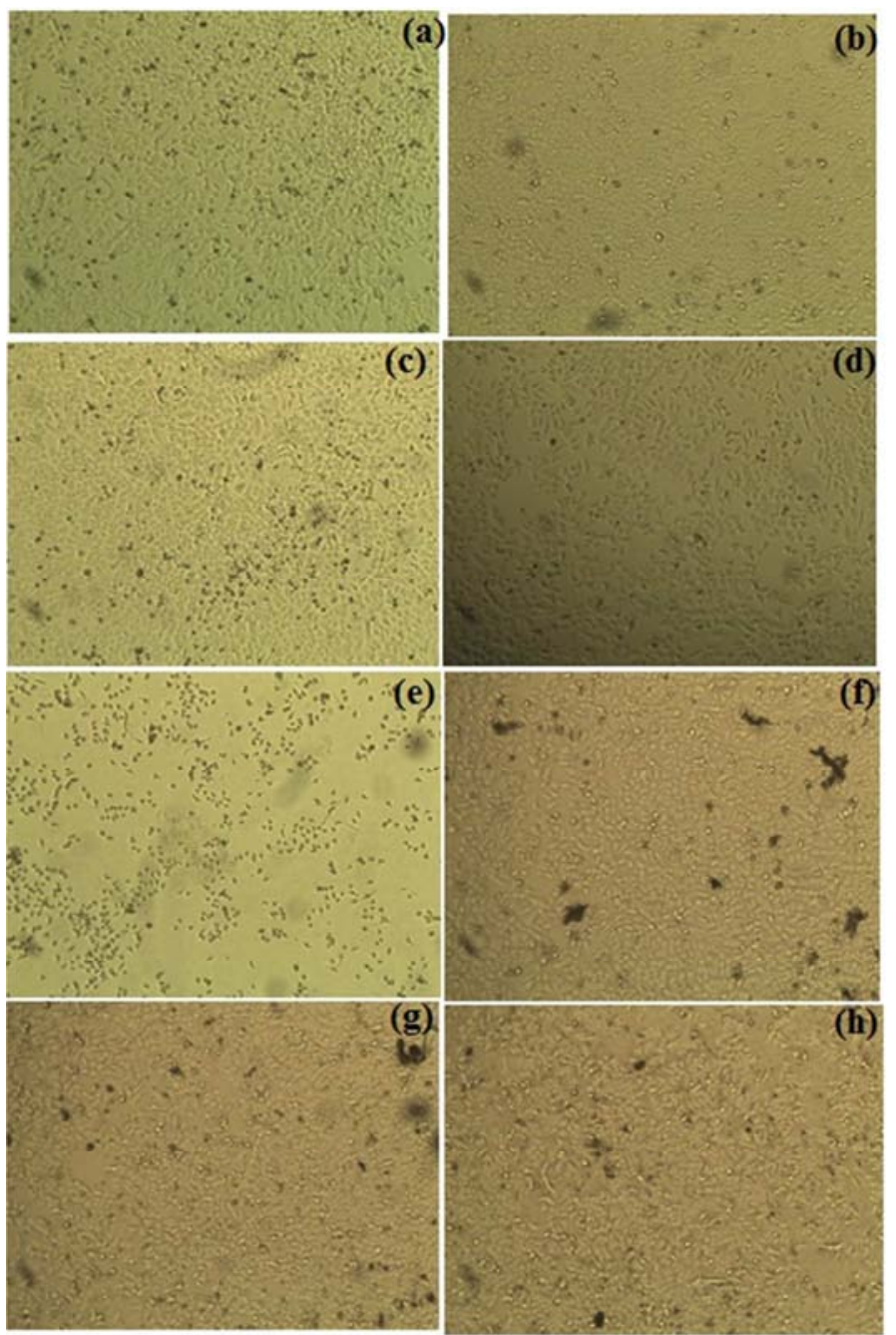

Figure 5. Cytotoxic effect of (a) control, (b) blank, (c) pure 2\% CMC, (d) CMC-gelatin composites (5:95), (e) CMC-gelatin composites (10:90), (f) CMCgelatin composites (15:85), (g) CMC-gelatin composites (20:80) and (h) CMC-gelatin composites (25:75). 
For biomedical application cytotoxicity is a major issue to concern about. The cytotoxic effect of CMC and CMCgelatin composites are presented in Figure 5. It was observed that all of the composites showed no cytotoxicity and in all cases survival of Hela cell is greater than $95 \%$. Among all of these composites, 2\% CMC has been chosen to make scaffold with gelatin considering the tensile strength.

\subsection{Antimicrobial Analysis}

The antimicrobial activity and quantitative test results of $\mathrm{CMC}$ and $\mathrm{CMC}$-gelatin composites are presented in Table-1. Gram negative bacteria, Escherichia coli and gram positive bacteria staphylococcus aureus were selected to assess susceptibility pattern. The zones of growth inhibition were compared with standard drug loaded disks. From the result of antimicrobial test it is clearly visualized that pure CMC and composites show larger inhibition zone against Staphylococcus aureus than E. coli.

Table 1. Antibacterial activity and quantitative measurement of inhibition zone of pure CMC and CMC-gelatin composites against gram positive and negative bacteria.

\begin{tabular}{|c|c|c|c|c|c|}
\hline & Microbial strain & CMC & Gel-CMC (85:15) & Gel-CMC (75:25) & Standard \\
\hline \multirow{2}{*}{ Antibacterial activity } & Staphylococcus aureus & + & + & + & 18 \\
\hline & Escherichia coli & + & + & + & 16 \\
\hline \multirow{2}{*}{ Quantitative measurement } & Staphylococcus aureus & 17 & 18. & 20.5 & \\
\hline & Escherichia coli & 10 & 16 & 19 & \\
\hline
\end{tabular}

\section{Conclusion}

The increasing demand of the biocomposites for the biomedical purposes leads to the responsibility and obligation of researchers to develop products with better properties compared with those of existing materials. The results reported in this study showed that CMC could be successfully added to gelatin for preparation of soft and elastic films that has good characteristics for tissue engineering application. The conclusion of the research can be summarized as (i) Homogeneous gelatin-CMC was successfully prepared and confirmed by FTIR, (ii) the surface of pure gelatin was less porous and uneven but CMC incorporation a much plainer texture with an incremented porosity has been observed and (iii) the composite films were more thermal stable, antibacterial activity and no cytotoxicity effect that suggest for its use as a wound dressing materials. CMC with natural polymer-based composite could endow many favorable properties such as hydrophilicity, biodegradability, biocompatibility, low cost and non-toxicity resulting in application of composites in skin tissue engineering.

\section{References}

[1] Madihally, S. V. and H. W. Matthew, Porous chitosan scaffolds for tissue engineering. Biomaterials, 1999. 20(12): p. 1133-1142.

[2] Agarwal, T., et al., Gelatin/Carboxymethyl chitosan based scaffolds for dermal tissue engineering applications. International journal of biological macromolecules, 2016. 93: p. 1499-1506.

[3] Maji, S., et al., Development of gelatin/carboxymethyl chitosan/nano-hydroxyapatite composite $3 D$ macroporous scaffold for bone tissue engineering applications. Carbohydrate Polymers, 2018.

[4] Chapekar, M. S., Tissue engineering: challenges and opportunities. Journal of Biomedical Materials Research Part A, 2000. 53(6): p. 617-620.

[5] Yin, Y., et al., Preparation and characterization of macroporous chitosan-gelatin/ $\beta$-tricalcium phosphate composite scaffolds for bone tissue engineering. Journal of Biomedical Materials Research Part A, 2003. 67(3): p. 844855 .

[6] Nie, W., et al., Three-dimensional porous scaffold by selfassembly of reduced graphene oxide and nano-hydroxyapatite composites for bone tissue engineering. Carbon, 2017. 116: p. 325-337.

[7] Baek, J., et al., Meniscal tissue engineering using aligned collagen fibrous scaffolds: comparison of different human cell sources. Tissue Engineering Part A, 2018. 24(1-2): p. 81-93.

[8] Kim, S., et al., Chitosan/gelatin-based films crosslinked by proanthocyanidin. Journal of Biomedical Materials Research Part B: Applied Biomaterials, 2005. 75(2): p. 442-450.

[9] Saravanan, S., et al., Scaffolds containing chitosan, gelatin and graphene oxide for bone tissue regeneration in vitro and in vivo. International journal of biological macromolecules, 2017. 104: p. 1975-1985.

[10] Ji, C. and J. Shi, Thermal-crosslinked porous chitosan scaffolds for soft tissue engineering applications. Materials Science and Engineering: C, 2013. 33(7): p. 3780-3785.

[11] Kong, L., et al., A study on the bioactivity of chitosan/nanohydroxyapatite composite scaffolds for bone tissue engineering. European Polymer Journal, 2006. 42(12): p. 3171-3179.

[12] Wang, G., et al., Preparation of cross-linked carboxymethyl chitosan for repairing sciatic nerve injury in rats. Biotechnology Letters, 2010. 32(1): p. 59.

[13] Bukzem, A. L., et al., Optimization of carboxymethyl chitosan synthesis using response surface methodology and desirability function. International journal of biological macromolecules, 2016. 85: p. 615-624.

[14] Hjerde, R. J. N., et al., Chemical composition of $O$ (carboxymethyl)-chitins in relation to lysozyme degradation rates. Carbohydrate Polymers, 1997. 34(3): p. 131-139. 
[15] Fiamingo, A. and S. P. Campana-Filho, Structure, morphology and properties of genipin-crosslinked carboxymethylchitosan porous membranes. Carbohydrate Polymers, 2016. 143: p. 155-163.

[16] Chen, X.-G. and H.-J. Park, Chemical characteristics of $O$ carboxymethyl chitosans related to the preparation conditions. Carbohydrate Polymers, 2003. 53(4): p. 355-359.

[17] Thi, T. T. H., et al., Oxidized cyclodextrin-functionalized injectable gelatin hydrogels as a new platform for tissueadhesive hydrophobic drug delivery. RSC Advances, 2017. 7(54): p. 34053-34062.
[18] Jorgensen, J. H. and J. D. Turnidge, Susceptibility Test Methods: Dilution and Disk Diffusion Methods*. 2015.

[19] Sheikholeslami, Z. S., et al., Exploring the effect of formulation parameters on the particle size of carboxymethyl chitosan nanoparticles prepared via reverse micellar crosslinking. Journal of microencapsulation, 2017. 34(3): p. 270-279.

[20] Wongkom, L. and A. Jimtaisong, Novel biocomposite of carboxymethyl chitosan and pineapple peel carboxymethylcellulose as sunscreen carrier. International journal of biological macromolecules, 2017. 95: p. 873-880. 